\title{
Tool use disorders after left brain damage
}

\author{
Josselin Baumard ${ }^{1}$, François Osiurak ${ }^{2}$, Mathieu Lesourd ${ }^{2}$ and Didier Le Gall ${ }^{1,3}$ \\ 1 Laboratoire de Psychologie des Pays de la Loire, Université d'Angers, Angers, France \\ 2 Laboratoire d'Etude des Mécanismes Cognitifs, Institut de Psychologie, Université Lyon 2, Bron, France \\ ${ }^{3}$ Unité de Neuropsychologie, Département de Neurologie, Centre Hospitalier Universitaire d'Angers, Angers, France
}

\author{
Edited by: \\ Cristina Massen, Leibniz Research \\ Centre for Working Environment \\ and Human Factors, Germany \\ Reviewed by: \\ Guido Gainotti, Policlinico Gemelli, \\ Italy \\ Tim Vanbellingen, University \\ Hospital Bern, Switzerland

\section{*Correspondence:} \\ Josselin Baumard, Laboratoire de \\ Psychologie des Pays de la Loire, \\ Maison des Sciences Humaines, \\ Université d'Angers, 5 bis, \\ Boulevard Lavoisier, 49045 Angers, \\ France \\ e-mail: josselin.baumard@ \\ univ-angers.fr; \\ François Osiurak, Laboratoire \\ d'Etude des Mécanismes Cognitifs, \\ Institut de Psychologie, Université \\ Lyon-2, 5 Avenue Pierre \\ Mendès-France, 69676 Bron, France \\ e-mail: francois.osiurak@ \\ univ-lyon2.fr
}

In this paper we review studies that investigated tool use disorders in left-brain damaged (LBD) patients over the last 30 years. Four tasks are classically used in the field of apraxia: Pantomime of tool use, single tool use, real tool use and mechanical problem solving. Our aim was to address two issues, namely, (1) the role of mechanical knowledge in real tool use and (2) the cognitive mechanisms underlying pantomime of tool use, a task widely employed by clinicians and researchers. To do so, we extracted data from 36 papers and computed the difference between healthy subjects and LBD patients. On the whole, pantomime of tool use is the most difficult task and real tool use is the easiest one. Moreover, associations seem to appear between pantomime of tool use, real tool use and mechanical problem solving. These results suggest that the loss of mechanical knowledge is critical in LBD patients, even if all of those tasks (and particularly pantomime of tool use) might put differential demands on semantic memory and working memory.

Keywords: apraxia, tool use, pantomime, mechanical problem solving, stroke

\section{INTRODUCTION}

Over the past century, a body of evidence has indicated that lesions in the left hemisphere can impair the ability to use tools, hereafter referred to as "apraxia of tool use." Nevertheless, there is neither consensus on the underlying cognitive processes (semantic knowledge about tool function, sensorimotor knowledge about tool manipulation, mechanical knowledge), nor on the way they are assessed (pantomime of tool use, single tool use, real tool use, mechanical problem solving). So, it may be difficult for students and researchers to obtain a comprehensive overview of tool use impairments after left brain damage. The major aim of this paper is to fill this gap by providing a synthesis of experimental results over the last 30 years. This will lead us to address two crucial issues: (1) The role of mechanical knowledge in tool use, which has received growing attention in recent years; (2) The cognitive processes supporting the most widely employed task, namely, pantomime of tool use.

Before discussing these issues directly, let us specify which studies are eligible for inclusion in the present review. Apraxia covers a wide range of disorders (e.g., constructive apraxia, gait apraxia, apraxia of speech, dressing apraxia) as well as several types of gestures (tool use, symbolic and meaningless gestures). However, we will only emphasize tool use impairment. Besides, the historical ideomotor/ideational apraxia dichotomy has been argued to be confusing, reflecting either a task-based or a process-based distinction (e.g., Hermsdörfer et al., 2006;
Osiurak et al., 2011; Lesourd et al., 2013a). Therefore, for the sake of clarity, we decided not to use this dissociation to select studies.

\section{TOOL USE ASSESSMENT}

Apraxia of tool use can be assessed in at least four ways depending on the amount of information given to patients.

\section{PANTOMIME OF TOOL USE}

Critical to this task is that patients are asked to demonstrate the use of tools without holding them in hand. The input modality may vary (visual presentation of the tool, verbal command, imitation) and the examiner may provide more or less information as to the name of the tool, its function, its usual corresponding object ${ }^{1}$ or the necessity of imagining holding the tool in hand. Imitation tasks can be performed without referring to tool knowledge, as in imitation of meaningless postures (Della Sala et al., 2006; see also Goldenberg, 1995, 1999; Goldenberg and Hagmann, 1997). Therefore, we did not consider results about imitation and we only included studies on pantomime of tool use on visual presentation and/or to verbal command.

\footnotetext{
${ }^{1}$ We shall use the terms tool and object to refer to the implement performing the action (e.g., screwdriver) and the recipient of the action (e.g., screw), respectively.
} 


\section{SINGLE TOOL USE}

Single tool use consists in demonstrating the use of a tool while holding it in hand but without the usual, corresponding object. Contrary to pantomimes, the tactile input is present, suggesting that patients do not need to form a mental representation of the tool. Additional information may be provided (name of the tool, the action or the goal of the action) but, for our purpose, we did not take these criteria into account.

\section{REAL TOOL USE}

In this task, patients are asked to actually use tools with the usual, corresponding object. We distinguished between two conditions (no-choice versus choice). In the no-choice condition, patients are presented with only the tool and its corresponding object. In the choice condition, several tools and objects are given. Two criteria can be found in the literature, namely, the presence/absence of tools/objects not useful for the action to be done (i.e., distractors) or the presence/absence of a sequence of at least two actions involving more than two tools/objects (i.e., multiple object task). This latter condition can be viewed as a choice condition since each time an action is performed with two tools/objects (e.g., striking the match on the matchbox), the remaining tools/objects (e.g., the candle) become distractors for this specific action.

\section{MECHANICAL PROBLEM SOLVING}

These tasks require using novel tools in order to solve an unfamiliar tool use situation (e.g., extracting a target from a box or lifting a cylinder). The solution can be found out from the mere observation of the device, perhaps without adopting trial-anderror strategy. This covers situations wherein familiar tools have to be used in a non-conventional way (e.g., screwing a screw with a knife). As for real tool use, two conditions exist: choice (i.e., selection of the correct tools among an array of novel tools) and no-choice (i.e., only the correct, novel tool is present).

\section{THEORETICAL BACKGROUND}

It is commonly assumed that tool use is supported by two systems: The conceptual and the production system. The role of the conceptual system is to form a mental, tool action representation. Three kinds of knowledge have been proposed in the literature. The first one corresponds to semantic knowledge about tool function, which contains information about the usual relationship between a familiar tool and its corresponding object or the context wherein it can be used (e.g., a hammer is commonly used with a nail and can be found in a workshop; Roy and Square, 1985; Rothi et al., 1991; Buxbaum, 2001). In other words, it refers to allocentric relationships (i.e., tool-object), and is associated with left anterior, temporal lobe lesions (Hodges et al., 2000; Goldenberg and Spatt, 2009; Goldenberg, 2013a).

Second, sensorimotor knowledge about tool manipulation comprises information about the movements associated with the usual manipulation of a specific tool (e.g., the use of a hammer requires ample elbow oscillations; Rothi et al., 1991; Buxbaum, 2001). So, contrary to semantic knowledge, sensorimotor knowledge is supposed to encode egocentric relationships (i.e., tooluser). Damage to the left inferior parietal lobe might impair this kind of knowledge (e.g., Buxbaum and Saffran, 2002; Buxbaum and Kalénine, 2010; Kalénine et al., 2013).

Third, mechanical knowledge provides information about relationships between the physical properties of tools and objects (e.g., hammering requires that the hammer is heavier than the nail; Goldenberg and Hagmann, 1998b; Goldenberg and Spatt, 2009; Osiurak et al., 2010, 2011, 2013; Osiurak, 2014). This kind of knowledge refers to allocentric relationships (i.e., tool-object) and might be also supported by the left inferior parietal lobe (Goldenberg and Spatt, 2009).

The role of the production system is to generate a specific movement pattern by taking into account both the environmental constraints and the tool action representation built by the conceptual system (for discussion, see Osiurak, 2013a,b). The dorsal stream would be the neural basis of this production system (Heilman et al., 1986; Buxbaum, 2001; Binkofski and Buxbaum, 2013).

The aforementioned kinds of knowledge have been suggested to be differentially involved depending on the given task (pantomime of tool use, single tool use, real tool use, mechanical problem solving). Special attention has to be paid to pantomime of tool use given that it might be grounded on processes that are not tool-specific. Indeed, the most widespread interpretation of impaired performance in this task stresses damage to sensorimotor knowledge (i.e., the sensorimotor knowledge hypothesis; Heilman et al., 1982; Buxbaum et al., 2005). However, it has also been hypothesized that it is a non-routine, creative task requiring working memory in order to temporarily maintain information about how the tool has to be held in hand and should be used with the corresponding, absent object (i.e., the working memory hypothesis; Roy and Hall, 1992; Bartolo et al., 2003). At last, pantomime of tool use has been assumed to be nothing else but a kind of symbolic gesture (i.e., the symbolic hypothesis; Goldenberg et al., 2003). In this view, the demonstration by pantomime would aim to communicate the idea of the action rather than to attempt to reproduce the gesture strictly speaking. We shall return to these three hypotheses in more detail below.

\section{METHODS}

The purpose of the present paper was to review the experimental data published on pantomime of tool use, single tool use, real tool use and mechanical problem solving since 1985 (i.e., the year Roy and Square published the conception-production model). To this end, several databases (i.e., PubMed, ScienceDirect, Eric, Francis, PBSC, Psycarticles, Web of Knowledge) were searched in 2013-2014 for the following keywords: "tool use," "object use," "apraxia," "limb apraxia," "ideational apraxia," "apraxia of tool use," and "stroke," "left brain damage," "left hemisphere."

\section{SELECTION OF PAPERS}

Only English language experimental studies were included. They had to meet the following criteria:

(1) Presence of right-handed patients with lesions confined to the left hemisphere. Studies were not included if they involved healthy subjects only or if they investigated disconnection syndromes. 
(2) Presence of a control group consisting of healthy subjects or at least non-neurological patients.

(3) Administration of at least one of the four critical tasks (i.e., pantomime of tool use, single tool use, real tool use, mechanical problem solving). Pantomime tasks had to be made of "pure" pantomime items, without other types of items such as symbolic gestures (e.g., waving goodbye). Besides, tasks were considered as mechanical problem solving tasks only if patients had to hold a tool to use with an object, and only if it could be achieved through inference rather than trial and error, so as to be comparable with other tool use tasks.

(4) Administration on verbal command, visual presentation or tactile input. Even though the aforementioned tasks can be administered on imitation, we did not consider this modality because imitation is not supposed to be accounted for by semantic knowledge about tool function, sensorimotor knowledge about tool manipulation or mechanical knowledge (see Roy and Square, 1985; Rothi et al., 1991). Moreover, there is no consistent correlation between production of symbolic gestures on verbal command and on imitation (Heath et al., 2001). Therefore, we focused on verbal, visual and tactile presentation of tools or objects. It is noteworthy that we could have studied modality effects, but we did not do so. Because of methodological heterogeneity in the field of apraxia, this would have led us to generate too many categories with very few studies for each modality, preventing us from drawing firm conclusions.

(5) Availability of quantitative behavioral data for both patients and controls, allowing us to convert mean performance levels into percentages, and to contrast them. Frequency of impairment among patients, $z$-scores, number of errors, and kinematic data were not taken into account. Finally, we excluded "redundant" studies (i.e., studies whose data had already been published) for it would have exaggerated some results.

Our keywords led us to create a corpus of 176 studies. Only 36 out of 176 studies fitted our criteria (see Figure 1). In this pool we counted 59 different tasks, considering that several studies included more than one relevant task. Regarding our criteria, tool use is frequently assessed through pantomime of tool use $(25 / 36,69 \%)$ whereas single tool use $(12 / 36,33 \%)$, real tool use
$(14 / 36,39 \%)$ and especially mechanical problem solving $(8 / 36$, $22 \%$ ) were only occasionally investigated over the last 30 years. This can be explained by a lack of consensus in this field (see Dovern et al., 2012).

\section{DATA EXTRACTION}

In many papers only apraxic left-brain damaged (LBD) patients are included, most often on the basis of imitation or pantomime tasks. However, although some manifestations of apraxia are more prevalent following left rather than right hemisphere lesions (Goldenberg, 2009), this is not the case for real tool use and naturalistic actions (Schwartz et al., 1999; Hartmann et al., 2005; Rumiati, 2005). We did not select these studies because our purpose was to analyze the consequences of left-brain-damage, rather than apraxia, on tool use. Indeed, if we did so, this would have led us to follow a pointless, circular reasoning, namely, apraxic patients are apraxic. Nevertheless, we reviewed these studies if they secondarily included non-apraxic LBD patients. In this case, we calculated the mean performance of apraxic and non-apraxic LBD patients (by taking into consideration, of course, the number of patients in each category). We acknowledge that this may be a bias since it does not display the performances of consecutive patients. However we believe it reflects the state of literature, and it prevented us from eliminating too many relevant studies.

\section{DATA ANALYSIS}

In order to make data from these 36 studies comparable, we converted mean performances and standard deviations into percentages. Then, we calculated the mean performance level for each task, weighted by sample sizes. Furthermore, for each study, we computed the difference between controls' scores minus LBD patients' scores (for a similar method, see Lesourd et al., 2013a,b). This procedure appears suitable for several reasons. First, given the low number of studies available and methodological heterogeneity, it was not relevant to conduct a meta-analysis. Second, the performances of control subjects can vary between studies, therefore focusing on differences rather than raw scores avoids a bias when comparing papers. At last, this procedure expresses the severity of the impairment in each task, which is a good way to determine whether different tasks call upon similar or different cognitive mechanisms (e.g., a difference of $50 \%$ in one task and $10 \%$ in another may lead us to infer divergent cognitive

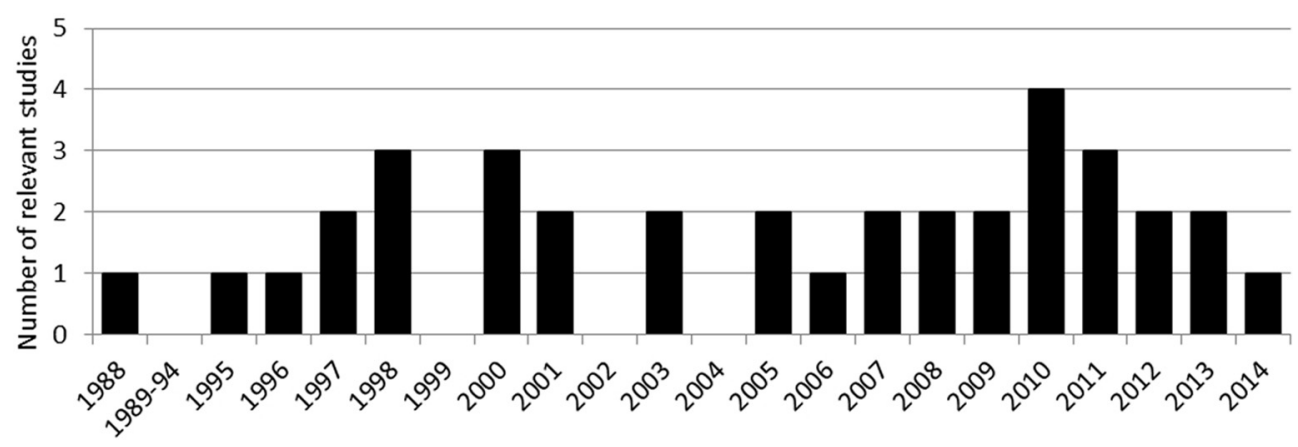

FIGURE 1 | Repartition of the 36 studies included in the present review over time. 
demands). However, results from this procedure have to be taken with caution in light of the high frequency of ceiling effects in control groups, which can artificially reduce the difference to patients.

\section{RESULTS}

\section{COMPARISONS BASED ON WEIGHTED MEANS}

As can be seen in Table 1, performance of LBD patients is lower for pantomime of tool use than for single and real tool use (mean scores $66,78,84 \%$, respectively; range $34-88,68-100,72-100$ ), with the two latter producing similar results at first sight. Actually, in terms of level of performance, pantomime of tool use is closer to mechanical problem solving (mean score 68\%, range 30-94), than to other conditions.

\section{COMPARISONS BASED ON GROUP DIFFERENCES}

Control-patient differences are presented in Figure 2 (raw scores are displayed in Table 1). Each circle corresponds to one task in one study. The Y-axis displays the distance in percentage between control subjects and LBD patients: The greater the difference, the

Table 1 | Performances of control subjects and LBD patients (mean scores and standard deviations).

\begin{tabular}{|c|c|c|c|c|c|c|c|c|c|}
\hline & \multirow[t]{2}{*}{ Patients (n) } & \multicolumn{2}{|c|}{$\begin{array}{l}\text { Pantomime of } \\
\text { tool use }\end{array}$} & \multicolumn{2}{|c|}{ Single tool use } & \multicolumn{2}{|c|}{ Real tool use } & \multicolumn{2}{|c|}{$\begin{array}{c}\text { Mechanical problem } \\
\text { solving }\end{array}$} \\
\hline & & NOR & LBD & NOR & LBD & NOR & LBD & NOR & LBD \\
\hline Flores-Medina et al., 2014 & 17 & $85(2)$ & $45(5)$ & - & - & - & - & - & - \\
\hline Hermsdörfer et al., 2013 & 23 & - & - & $99(1)$ & $71(27)$ & - & - & - & - \\
\hline Jarry et al., 2013 & 16 & $87(11)$ & $47(36)$ & $93(9)$ & $72(27)$ & $98,8(3)$ & $76(29)$ & $92(10)$ & $58(33)$ \\
\hline Bickerton et al., 2012 & 74 & - & - & - & - & $96(7)$ & $80(32)$ & - & - \\
\hline Hogrefe et al., 2012 & 24 & $92(7)$ & $69(27)$ & - & - & - & - & - & - \\
\hline Poole et al., 2011 & 30 & - & - & - & - & $88(8)$ & $76(7)$ & - & - \\
\hline Papeo et al., 2011 & 12 & - & - & $96(1)$ & $85(4)$ & - & - & - & - \\
\hline Randerath et al., 2011 & 25 & $100(7)$ & $75(34)$ & $100(0)$ & $88(16)$ & $100(0)$ & $100(2)$ & - & - \\
\hline Randerath et al., 2010 & 42 & - & - & $100(0)$ & 79 (19) & - & - & - & - \\
\hline Stamenova et al., 2010 & 42 & $95(1)$ & $71(4)$ & - & - & - & - & - & - \\
\hline Vanbellingen et al., 2010 & 84 & $88(12)$ & $58(32)$ & - & - & - & - & - & - \\
\hline Dawson et al., 2010 & 6 & $95(5)$ & $85(10)$ & - & - & - & - & - & - \\
\hline Jacobs et al., 2009 & 18 & - & - & $94(4)$ & $69(28)$ & - & - & - & - \\
\hline Osiurak et al., 2009 & 20 & - & - & - & - & $100(2)$ & 89 (19) & $85(7)$ & $64(20)$ \\
\hline Lunardelli et al., 2008 & 30 & - & - & - & - & - & - & $45(24)$ & $30(17)$ \\
\hline Osiurak et al., 2008 & 16 & $93(6)$ & $71(30)$ & - & - & $99(2)$ & $92(14)$ & - & - \\
\hline Goldenberg et al., 2007* & 11 & 93 & 80 & - & - & 95 & 83 & 100 & 94 \\
\hline Bartolo et al., 2007 & 5 & $92(4)$ & $44(33)$ & - & - & $91(7)$ & $74(12)$ & $98(3)$ & 81 (19) \\
\hline Jax et al., 2006 & 15 & $91(6)$ & $81(13)$ & - & - & - & - & - & - \\
\hline Buxbaum et al., 2005 & 13 & $89(1)$ & 71 (19) & - & - & - & - & - & - \\
\hline Hartmann et al., 2005 & 25 & $93(1)$ & $66(5)$ & - & - & $92(2)$ & $83(3)$ & $99(1)$ & $88(3)$ \\
\hline Goldenberg et al., 2003 & 40 & $96(3)$ & $66(27)$ & - & - & - & - & - & - \\
\hline Bartolo et al., 2003 & 1 & $97(5)$ & 60 & $100(0)$ & 100 & - & - & - & - \\
\hline Halsband et al., 2001 & 13 & 98 & 80 & - & - & 100 & 98 & - & - \\
\hline Hanna-Pladdy et al., 2001 & 14 & 85 & 41 & - & - & - & - & - & - \\
\hline Neiman et al., 2000 & 30 & - & - & - & - & 98 & 78 & - & - \\
\hline Cubelli et al., 2000 & 19 & - & - & 93 & $72(28)$ & - & - & - & - \\
\hline Roy et al., 2000 & 46 & $93(3)$ & $87(8)$ & - & - & - & - & - & - \\
\hline Goldenberg and Hagmann, 1998b & 42 & 84 & 50 & - & - & 99 & 92 & 100 & 85 \\
\hline Goldenberg and Hagmann, 1998a & 35 & $86(11)$ & $34(32)$ & $99(3)$ & $78(21)$ & - & - & - & - \\
\hline Roy et al., 1998 & 26 & $95(3)$ & $88(4)$ & - & - & - & - & - & - \\
\hline Heilman et al., 1997 & 21 & $86(23)$ & $56(27)$ & $94(12)$ & $68(23)$ & $100(0)$ & $83(17)$ & $82(17)$ & $57(25)$ \\
\hline Schnider et al., 1997 & 16 & $98(2)$ & $78(21)$ & $100(0)$ & $93(10)$ & - & - & - & - \\
\hline Belanger and Duffy, 1996 & 25 & $91(5)$ & $71(14)$ & $90(3)$ & 77 (12) & - & - & - & - \\
\hline Foundas et al., 1995 & 10 & - & - & - & - & $100(0)$ & $72(22)$ & - & - \\
\hline Barbieri and De Renzi, 1988 & 56 & $97(4)$ & $76(23)$ & - & - & - & - & - & - \\
\hline Weighted mean & & 92 & 66 & 97 & 77 & 97 & 84 & 85 & 68 \\
\hline Minimum mean score & & 84 & 34 & 90 & 68 & 88 & 72 & 45 & 30 \\
\hline Maximum mean score & & 100 & 88 & 100 & 100 & 100 & 100 & 100 & 94 \\
\hline
\end{tabular}

*We included this paper although some data have already been published in a larger sample (Hartmann et al., 2005).

Bolded values are non-significant differences. 


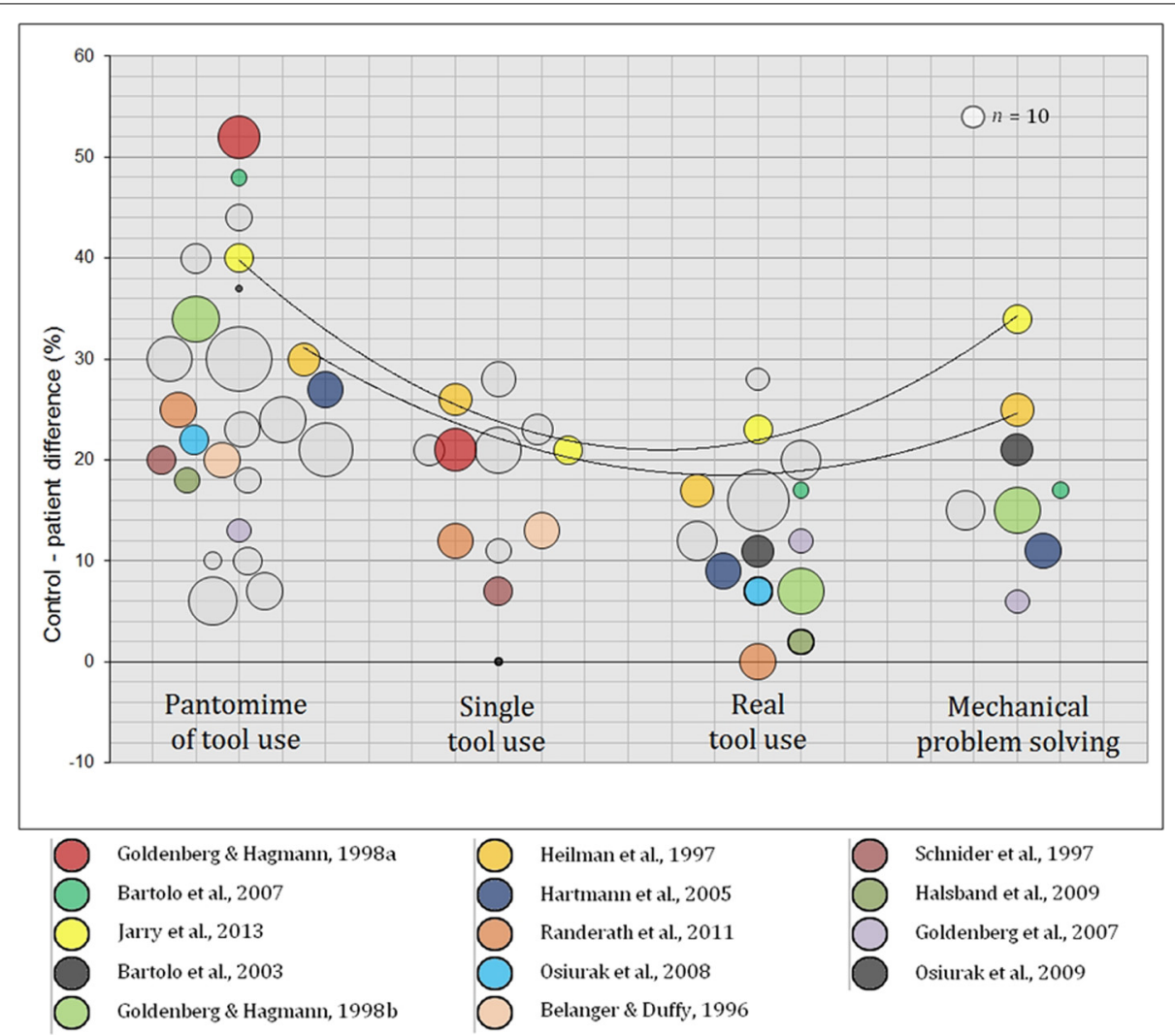

FIGURE 2 | Differences (in percentage) between control subjects and LBD patients: pantomime of tool use, single tool use, real tool use and mechanical problem solving. Colored circles correspond to studies that investigated more than one task. Circles in bold are non-significant differences. Curves were drawn for studies that investigated the four tasks. higher the impairment. Colors were assigned to some circles in order to stress studies in which two, three or four tasks were administered. Gray circles represent studies that investigated only one of the four tasks. Circles in bold are non-significant differences. Circle surfaces express sample sizes and curves were drawn for the only two studies that investigated all of the four tasks (Heilman et al., 1997; Jarry et al., 2013).

At first sight, there is more discrepancy between studies concerning pantomime of tool use, and there is a gradient from pantomime of tool use (mean difference $25 \%$, range 6-52) to real tool use (mean 13\%, range 0-28) with single tool use being intermediary (mean 17\%, range 0-28). Actually, the first is systematically more difficult than the two latter if we focus on studies in which at least two tasks were administered (i.e., colored circles in Figure 2).

Second, this gradient is less obvious between single and real tool use. Although previous studies found no significant association between these tasks (Butler, 2002; Bickerton et al., 2012; see also Riddoch et al., 1989), only two studies investigated both of these tasks in respect of our criteria: According to Jarry et al. (2013), both of them are equally difficult whereas Randerath et al. (2011) found real tool use to be much easier. This gap is most likely due to methodological variations since the latter authors employed a very structured task (i.e., patients were assessed with only two items, they did not have to select tools in real tool use and they were provided with verbal information about the action to be done).

Finally mechanical problem solving appears to be the most difficult task after pantomime of tool use (mean difference $18 \%$, range 6-34). More specifically, the control-patient difference is almost always greater than in single and/or real tool use. Only one out of eight studies reported the opposite finding in LBD patients with posterior lesions only (Goldenberg et al., 2007). Nevertheless, real tool use and mechanical problem solving are assessed with a wide array of tasks. In light of these results, it appeared necessary to control for this methodological discrepancy.

\section{EFFECT OF CHOICE AND DISTRACTORS IN REAL TOOL USE AND MECHANICAL PROBLEM SOLVING}

We divided data from real tool use and mechanical problem solving into two categories: In the no-choice condition, patients are presented with only one tool and its corresponding object (e.g., a match and a matchbox) whereas in the choice condition, they are presented with three or more tools/objects (e.g., a match, a matchbox and a candle; also referred as to multiple object tasks). Within this latter condition, studies were also distinguished by the presence/absence of distractors, defined as tools/objects not useful for the task to be done (e.g., a match, a matchbox and a hammer). Situations in which at least two tasks are presented 
simultaneously (e.g., making coffee, fixing a tape recorder) were judged to include distractors since tools that are useful for one task are useless for the other.

As can be seen in Table 2 and Figure 3, only two studies investigated both choice and no choice in both real tool use and mechanical problem solving (Heilman et al., 1997; Jarry et al., 2013). Overall, although mechanical problem solving is more difficult than real tool use, these tasks produce similar results in that reducing the number of tools/objects enhances performances in both conditions. The only study that investigated real tool use (choice) without distractors (Neiman et al., 2000) found similar results.

Finally, as shown in Table 3, pantomime of tool use is more difficult than single tool use, which is more difficult than real tool use (no choice), with choice conditions being intermediary between pantomime of tool use and single tool use. Nevertheless, it is noteworthy that even in no choice condition, LBD patients' performance is significantly impaired as compared to controls.

\section{ASSOCIATIONS BETWEEN TASKS}

We intended to determine whether associations can be found between the tasks of interest. However, given that too few studies explored more than one condition, we only described association tendencies. To this end, we displayed control-patient differences from each study in which at least two tasks where investigated, among pantomime of tool use, single tool use, real tool use and mechanical problem solving (see Figure 4).

As can be seen, stronger positive associations were found between pantomime of tool use, real tool use and mechanical problem solving than between single tool use, real tool use and mechanical problem solving. A negative association was observed between single tool use and mechanical problem solving, but this observation has to be taken with caution given that it concerned only two studies. Interestingly, a slight impairment in mechanical problem solving coincides with more substantial impairment in pantomime of tool use than in real tool use. Furthermore, there is a positive association between mechanical problem solving and pantomime of tool use.

\section{DISCUSSION}

The aim of the present paper was to provide an overview of tool use impairments after left brain damage. More precisely, we shall discuss the role of mechanical knowledge in tool use as well as the cognitive mechanisms supporting pantomime of tool use.

\section{THE ROLE OF MECHANICAL KNOWLEDGE IN TOOL USE}

Three kinds of conceptual knowledge have been proposed to support real tool use: Semantic knowledge about tool function (Roy and Square, 1985; Rothi et al., 1991; Buxbaum, 2001), sensorimotor knowledge about tool manipulation (Rothi et al., 1991; Buxbaum, 2001) and mechanical knowledge about the physical properties of tools and objects (Goldenberg and Hagmann, 1998b; Goldenberg and Spatt, 2009; Osiurak et al., 2010, 2011). We shall address these hypotheses in turn.

Semantic knowledge provides individuals with information about the usual relationship between familiar tools and objects (e.g., a hammer is usually used with a nail). Therefore, it might be required in at least four situations: When matching pictures of tools with the corresponding, usual object (e.g., hammer/nail) or the context in which they can be used (e.g., hammer/workshop); when it is necessary to select tools/objects to be used together; when pantomiming the use of tools; and when performing single tool use. Indeed, given that objects are not present in the two latter situations, access to semantic knowledge is necessary to produce the right conventional action (e.g., hammering is relevant with a nail but not with a shoe). Interestingly, patients with semantic dementia, who have lost semantic knowledge about tools, have been demonstrated to perform better in no-choice situations and mechanical problem solving, suggesting that these tasks put less demands on functional knowledge (Hodges et al., 2000; Bozeat et al., 2002; Silveri and Ciccarelli, 2009).

In our data, LBD patients perform better in real tool use (no choice; mean control-patient difference $8 \%$, range $0-16$ ) than in pantomime of tool use $(25 \%, 6-52)$ and single tool use $(17 \%, 0$ 28 ). In other words, the more contextual information patients receive, the better they perform. Presumably, this contextual advantage may be a semantic advantage in that the presence of objects in real tool use provides sufficient information and makes retrieval from semantic memory unnecessary. Furthermore, the choice condition of real tool use $(18 \%, 9-28)$ is more difficult than the no-choice condition of real tool use. These results are consistent with the semantic hypothesis. Nevertheless, patients still perform worse than controls in real tool use (no choice) and mechanical problem solving $(18 \%, 6-34)$. As a consequence, disruption of semantic knowledge accounts for some, but not all, of tool use impairments. In other words, this kind of knowledge is not sufficient to support tool use (see also Buxbaum et al., 1997).

Sensorimotor knowledge links specific movements to specific tools (e.g., using a hammer requires ample elbow oscillations). Three predictions can be derived from this hypothesis. First, this kind of knowledge should be necessary in any task involving the production of tool-related movements, among which are pantomime of tool use, single tool use and real tool use. Second, choice situations should not be more difficult than no-choice situations because the same movement is required in both cases (e.g., hammering does not vary depending on the number of tools on the desk). Third, the loss of sensorimotor knowledge should not interfere in the use of novel tools, such as in mechanical problem solving.

Our results do not confirm these predictions. Indeed, LBD patients are not impaired to a similar extent in pantomime of tool use $(25 \%)$, single tool use $(17 \%)$, and real tool use (no-choice, $8 \%$ ). Moreover, the choice condition of real tool use is more difficult than the no-choice condition of real tool use even though this dissociation has been assessed in only two studies (Heilman et al., 1997; Jarry et al., 2013) and remains to be confirmed. At last, the sensorimotor hypothesis does not account for impaired performance of LBD patients in mechanical problem solving. On these accounts, experimental data did not prove that apraxia of tool use in LBD patients is due to the loss of sensorimotor knowledge.

Finally, mechanical knowledge about the physical properties of tools and objects (e.g., hammering requires that the hammer is heavier than the nail) may be necessary to use both familiar and novel tools, and might be supported by the left inferior parietal 


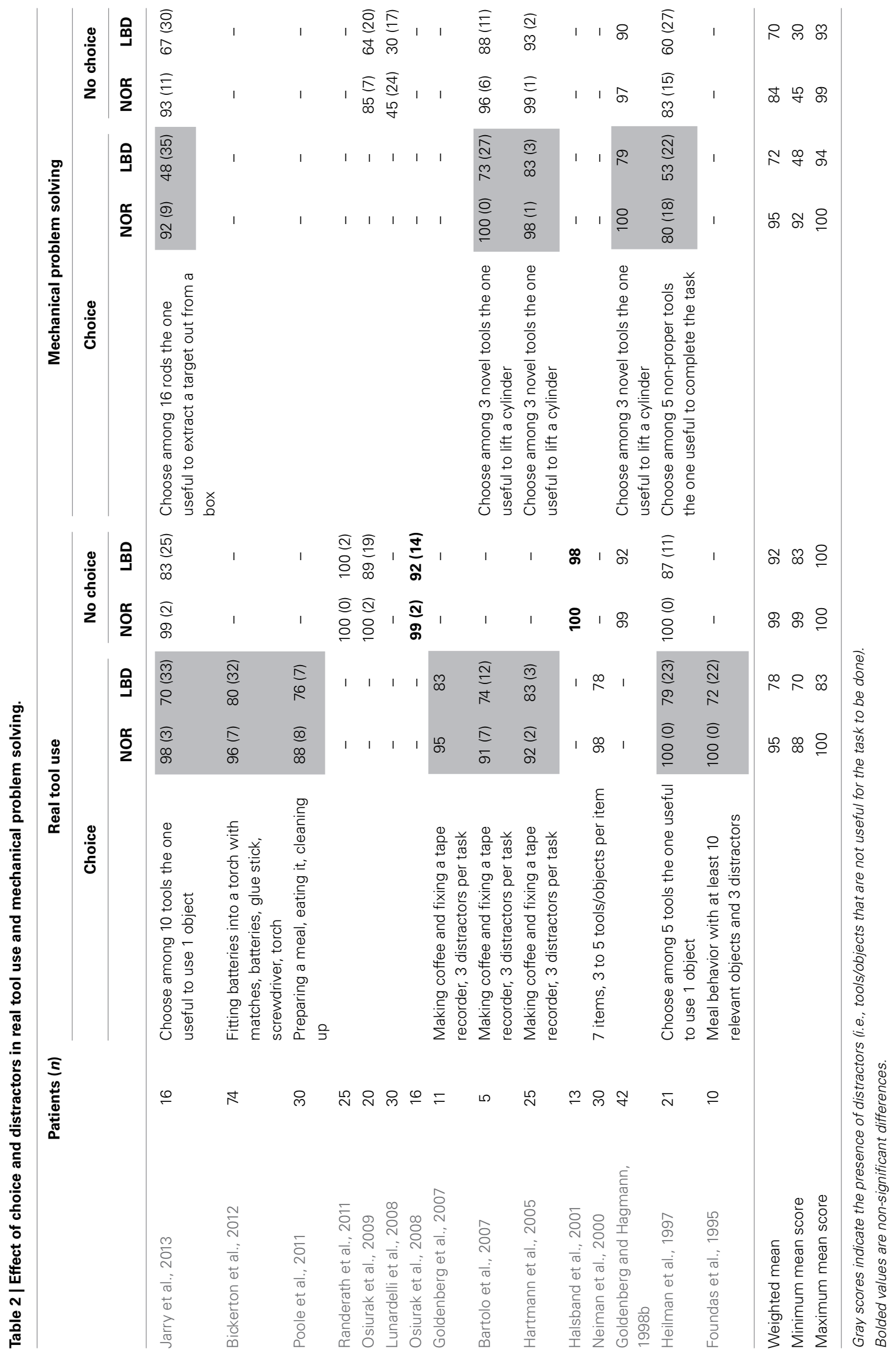




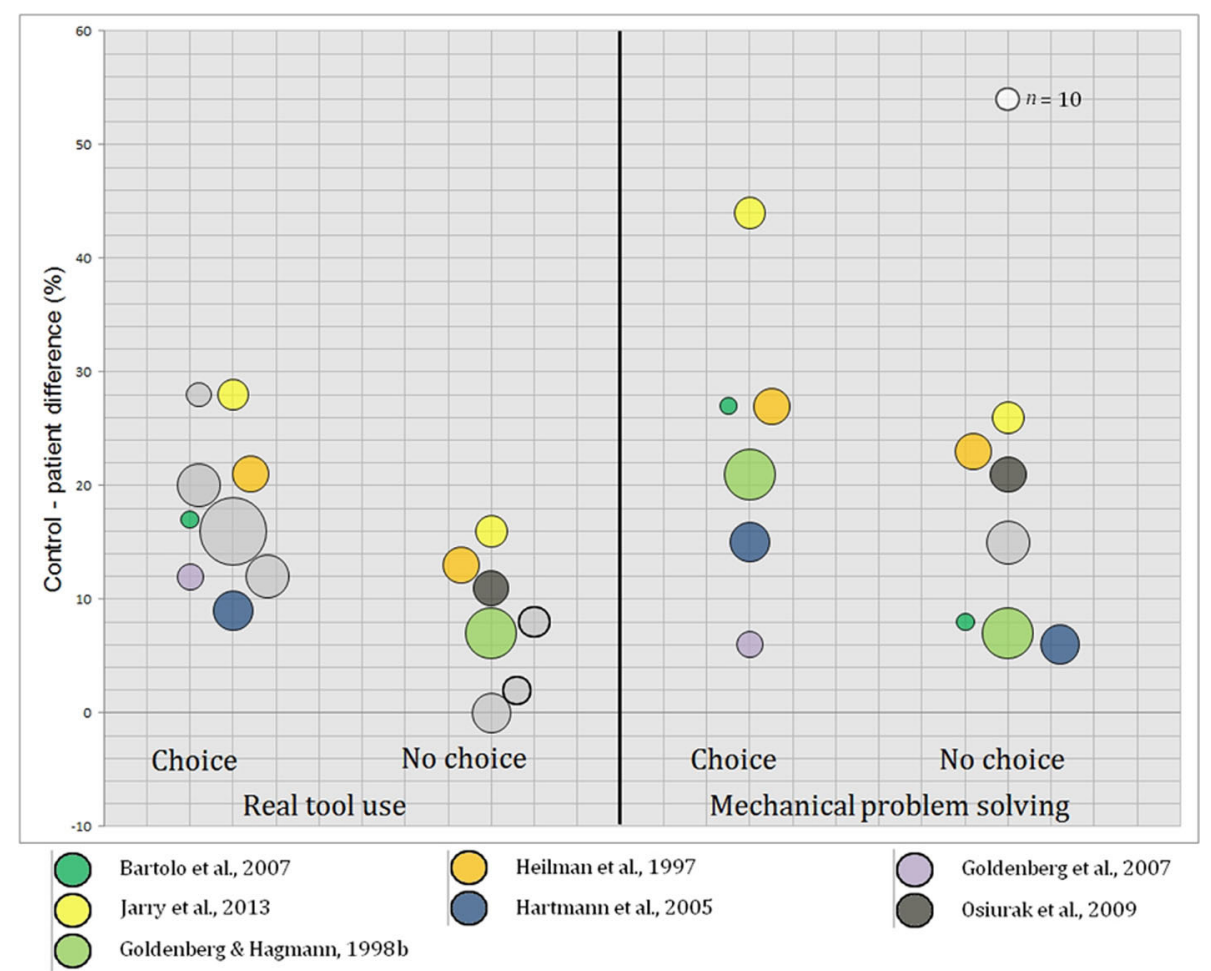

FIGURE 3 | Differences (in percentages) between control subjects and LBD patients in real tool use (Choice and No-Choice) and mechanical problem solving (Choice and No-Choice). Colored circles correspond to studies that investigated more than one condition. Circles in bold are non-significant differences.

Table 3 | Mean control-patient differences.

\begin{tabular}{lcc}
\hline & $\begin{array}{c}\text { Mean control-patient } \\
\text { difference (\%) }\end{array}$ & Range \\
\hline Pantomime of tool use & 25 & $6-52$ \\
Single tool use & 17 & $0-28$ \\
Mechanical problem solving (no choice) & 15 & $6-26$ \\
Real tool use (no choice) & 8 & $0-16$ \\
Mechanical problem solving (choice) & 23 & $6-44$ \\
Real tool use (choice) & 18 & $9-28$ \\
\hline
\end{tabular}

lobe (Goldenberg and Spatt, 2009). So, LBD patients are supposed to be concurrently impaired in both of these tasks.

Overall, our results confirmed this prediction (real tool use, mean control-patient difference $13 \%$; mechanical problem solving: 18\%). Moreover, LBD patients are constantly impaired in mechanical problem solving and, in studies that investigated both conditions, failure to solve mechanical problems was systematically associated with failure to use familiar tools (Heilman et al., 1997; Goldenberg and Hagmann, 1998b; Hartmann et al., 2005; Bartolo et al., 2007; Goldenberg et al., 2007; Osiurak et al., 2009; Jarry et al., 2013). Additionally, as shown in Figure 4, there is a clear positive association between the two tasks. These results lead us to suggest that mechanical knowledge is necessary to use familiar tools and objects.

To conclude, experimental data obtained over the last 30 years indicate that real tool use might be supported by at least two kinds of knowledge, both of them referring to allocentric relationships: Semantic knowledge about tool function and mechanical knowledge. These two types of knowledge might partially compensate for each other, in that studies on semantic dementia already described impaired use of familiar tools in the context of preserved mechanical problem solving (Hodges et al., 2000; Bozeat et al., 2002; Silveri and Ciccarelli, 2009). However, to our knowledge, this pattern has never been found in LBD patients. On the other hand, the reverse pattern (i.e., impaired, but better performance in real tool use than in mechanical problem solving; see Table 1) was frequently observed, suggesting that loss of mechanical knowledge can be partially compensated by intact semantic knowledge although it is critical to account for tool use disorders in LBD patients. We shall now discuss the cognitive processes underlying pantomime of tool use.

\section{THE COGNITIVE PROCESSES SUPPORTING PANTOMIME OF TOOL USE}

Three hypotheses have been proposed to explain the cognitive basis of pantomime of tool use: The sensorimotor knowledge hypothesis (Heilman et al., 1982; Buxbaum et al., 2005), the symbolic hypothesis (Goldenberg et al., 2003) and the working memory hypothesis (Roy and Hall, 1992; Bartolo et al., 2003).

According to the sensorimotor knowledge hypothesis, pantomime of tool use requires individuals to implicitly recover gesture representations that contain invariant, egocentric relationships, and that are specific to particular tools. Therefore, as suggested above, there should be no difference between 


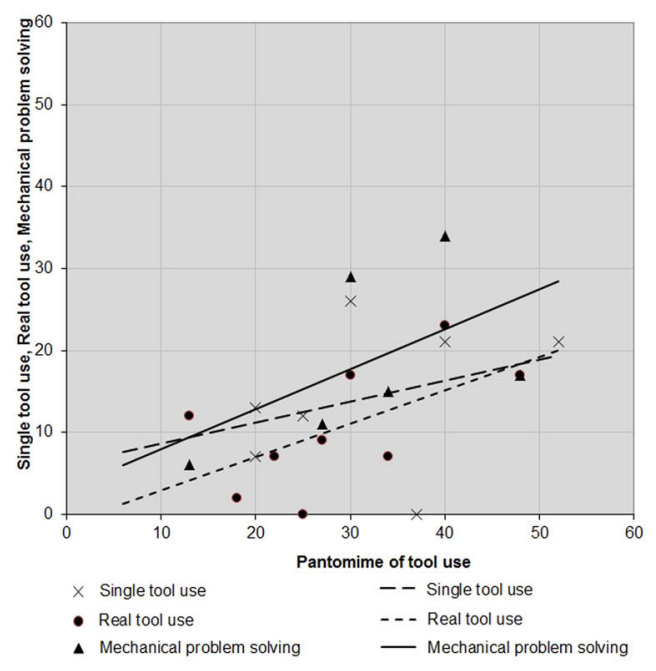

FIGURE 4 | Associations between pantomime of tool use and single tool use, real tool use, and mechanical problem solving (left panel) and between mechanical problem solving and pantomime of tool

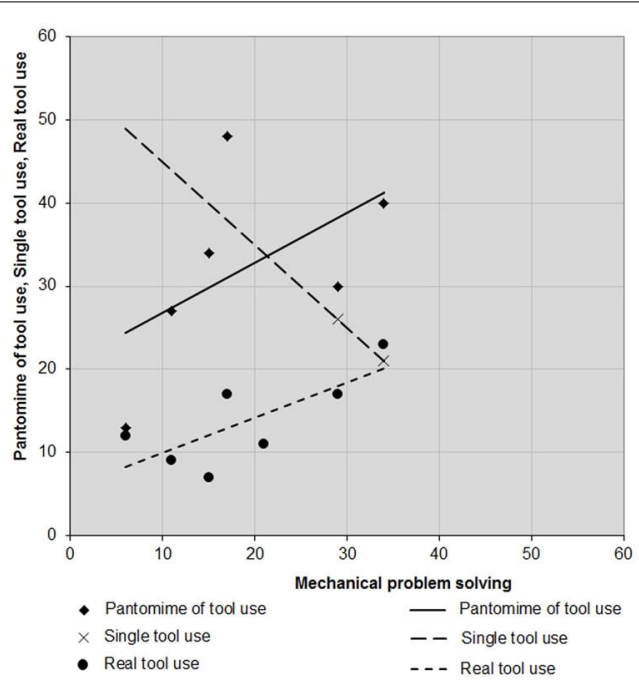

use, single tool use and real tool use (right panel). Each point corresponds to control-patient differences. Slopes illustrate the degree of association. pantomime of tool use, single tool use and real tool use. Indeed, because these representations are egocentric and invariant, the presence/absence of tools and objects should not modify controlpatient differences. However, the present review confirmed that pantomime of tool use is much more difficult than real tool use (see also Riddoch et al., 1989; Roy and Hall, 1992; Butler, 2002; Bartolo et al., 2003; Bickerton et al., 2012). Moreover, pantomime of tool use seems to be poorly associated with single tool use compared with real tool use and even mechanical problem solving (see Figure 4). These results thus do not favor the sensorimotor knowledge hypothesis.

The symbolic hypothesis assumes that defective pantomime of tool use is due to asymbolia, that is, a "general inability to express concepts by means of learned signs" (Goldenberg et al., 2003). As an example, drawing from memory implies to select typical features of the object to be drawn (e.g., the shape of both the handle and head of a hammer). Presumably, asymbolia should impair any activity that requires access to semantic memory, such as language, drawing from memory and pantomime of tool use (see Goldenberg, 2013b). Indeed, this hypothesis also presumes that pantomimes are part of communicative gestures in that they require patients to select distinctive features of the sensory appearance of absent tools/objects (e.g., the shape of the handle of a hammer) and to abstract properties that do not contribute to recognizability (e.g., the color or the material of the handle) in order to produce a canonical, recognizable gesture.

So, pantomime of tool use should be more difficult than single tool use since in the latter, patients do not need to communicate the idea of the tool because they already handle it. Our data are consistent with this hypothesis: Pantomime of tool use (mean control-patient difference 25\%) and single tool use (17\%) appear to be weakly associated and the first is consistently more difficult than the latter over studies. Nevertheless, pantomime of tool use is closer to mechanical problem solving than to single tool use (see
Table 3, Figures 3, 4) and in previous studies, asymbolia alone could not account for pantomime disturbances in LBD patients (Goldenberg et al., 2003). Further research is thus required on this point.

In line with the working memory hypothesis, pantomiming the use of tools leads individuals to form a mental representation of the tool in hand, the object on the desk and the action to be performed. Once this layout has been imagined, it has to be maintained in working memory until the gesture is finished. This implies that holding a tool in hand and/or seeing the object provides cues, hence reducing the degrees of freedom and so the number of possible errors (Roy and Hall, 1992; Bartolo et al., 2003). As a consequence, the presence of actual tools reduces the load on working memory and enhances performance. The gradient we already described is consistent with this hypothesis.

To sum up, the present review found the working memory hypothesis and, to a lesser extent, the symbolic hypothesis, to be most relevant as regards pantomime of tool use. On the other hand, the sensorimotor knowledge hypothesis remains to be demonstrated. Another key finding is the similar difficulty level and the relationship between mechanical problem solving and pantomime of tool use (see Table 3 and Figure 4). Previous studies reported significant correlations between these tasks (Heilman et al., 1997; Goldenberg and Hagmann, 1998b; Jarry et al., 2013). This finding is not compatible with cognitive models of apraxia (Roy and Square, 1985; Rothi et al., 1991; Buxbaum, 2001) but rather suggests that pantomime of tool use is a composite task that may call for mechanical knowledge, in addition with semantic knowledge and working memory. In fact, this task can be viewed as a kind of problem solving for it may require forming a mental representation through identification and combination of distinctive features of tools and actions (see Goldenberg et al., 2003; Goldenberg, 2009) or, put differently, technical means and technical ends (Osiurak et al., 2010, 2011). 
Table 4 | Cognitive demands depending on the task.

\begin{tabular}{|c|c|c|c|c|}
\hline & $\begin{array}{c}\text { Pantomime of } \\
\text { tool use }\end{array}$ & $\begin{array}{l}\text { Single } \\
\text { tool use }\end{array}$ & $\begin{array}{l}\text { Real tool } \\
\text { use }\end{array}$ & $\begin{array}{l}\text { Mechanical } \\
\text { problem } \\
\text { solving }\end{array}$ \\
\hline $\begin{array}{l}\text { Semantic knowledge } \\
\text { about tool function } \\
\text { and context }\end{array}$ & + & + & + & - \\
\hline $\begin{array}{l}\text { Mechanical knowledge } \\
\text { about physical properties } \\
\text { of tools/objects }\end{array}$ & + & + & + & ++ \\
\hline Working memory & ++ & + & - & - \\
\hline Production system & + & + & + & + \\
\hline
\end{tabular}

Before concluding, let us discuss results indicating differences between choice and no choice conditions. On the whole, the presence of numerous tools seems to be a major obstacle to LBD patients but not to control subjects. Note that although this finding is intuitive, the cognitive models of apraxia (Roy and Square, 1985; Rothi et al., 1991; Buxbaum, 2001) do not address the issue of how humans choose tools and objects. Interestingly, the choice effect is true for familiar as well as novel tools and, as a result, questions the relationship between mechanical knowledge and tool substitutions. Unfortunately, only one of the selected studies investigated real tool use (choice) without distractors (Neiman et al., 2000). Consequently, it remains unknown whether LBD patients fail multiple object tasks because of a planning impairment, interference from distractors or inability to select and combine useful/useless tools. Nevertheless, these results remain to be confirmed because ceiling effects prevented us from computing the real difference between choice and no-choice conditions in control group. Therefore, such a difference among patients could be accounted for by the intrinsic difficulty of choice conditions. Future research is needed to disentangle the origin of the choice effect in LBD patients.

\section{CONCLUSION}

To conclude, pantomime of tool use, single tool use, real tool use and mechanical problem solving seem to have at least one cognitive mechanism in common, which may be the ability to retrieve mechanical knowledge on the basis of identification and combination of distinctive features of tools and objects. Nevertheless, each task calls for differential demands depending on presence/absence, familiarity/novelty and number of tools/objects (see Table 4). This theoretical distribution challenges the idea that tool use in general, and pantomime of tool use in particular call for sensorimotor knowledge. Note also that data reported here focus on left brain damage but do not exclude a role of the right hemisphere in tool use (Schwartz et al., 1999; Hartmann et al., 2005; Rumiati, 2005). In sum, although apraxia of tool use is classically viewed as a disorder of movement representations/motor control, the present review emphasizes that apraxia of tool use in LBD patients may be first and foremost a cognitive disorder involving the understanding of how tools and objects have to be used together (Osiurak et al., 2010, 2011; Goldenberg, 2013a).

\section{ACKNOWLEDGMENTS}

This work was supported by grants from ANR (Agence Nationale pour la Recherche; Project Démences et Utilization d'Outils/Dementia and Tool Use, NANR 2011 MALZ 006 03), and was performed within the framework of the LABEX CORTEX (ANR-11-LABX-0042) of Université de Lyon, within the program "Investissements d'Avenir" (ANR-11- IDEX-0007) operated by the French National Research Agency (ANR).

\section{SUPPLEMENTARY MATERIAL}

The Supplementary Material for this article can be found online at: http://www.frontiersin.org/journal/10.3389/fpsyg.2014. 00473/abstract

\section{REFERENCES}

*Barbieri, C., and De Renzi, E. (1988). The executive and ideational components of apraxia. Cortex 24, 535-543. doi: 10.1016/S0010-9452(88)80047-9

*Bartolo, A., Cubelli, R., Della Sala, S., and Drei, S. (2003). Pantomimes are special gestures which rely on working memory. Brain Cogn. 53, 483-494. doi: 10.1016/S0278-2626(03)00209-4

*Bartolo, A., Daumüller, M., Della Sala, S., and Goldenberg, G. (2007). Relationship between object-related gestures and the fractionated object knowledge system. Behav. Neurol. 18, 143-147. doi: 10.1155/2007/241670

*Belanger, S. A., and Duffy, R. J. (1996). The assessment of limb apraxia: an investigation of task effects and their cause. Brain Cogn. 32, 384-404. doi: 10.1006/brcg.1996.0072

*Bickerton, W., Riddoch, M. J., Samson, D., Bahrami Balani, A., Mistry, B., and Humphreys, G. W. (2012). Systematic assessment of apraxia and functional predictions from the Birmingham Cognitive Screen. J. Neurol. Neurosur. Psychiatry 83, 513-521. doi: 10.1136/jnnp-2011-300968

Binkofski, F., and Buxbaum, L. J. (2013). Two action systems in the human brain. Brain Lang. 127, 222-229. doi: 10.1016/j.bandl.2012.07.007

Bozeat, S., Lambon Ralph, M. A., Patterson, K., and Hodges, J. R. (2002). When objects lose their meaning: what happens to their use? Cogn. Affect. Behav. Neurosci. 2, 236-251. doi: 10.3758/CABN.2.3.236

Butler, J. A. (2002). How comparable are tests of apraxia? Clin. Rehabil. 16, 389-398. doi: 10.1191/0269215502cr493oa

Buxbaum, L. J. (2001). Ideomotor apraxia: a call to action. Neurocase 7, 445-488. doi: 10.1093/neucas/7.6.445

*Buxbaum, L. J., Johnson-Frey, S. H., and Bartlett-Williams, M. (2005). Deficient internal models for planning hand-object interactions in apraxia. Neuropsychologia 43, 917-929. doi: 10.1016/j.neuropsychologia.2004.09.006

Buxbaum, L. J., and Kalénine, S. (2010). Action knowledge, visuomotor activation, and embodiment in the two action systems. Ann. N.Y. Acad. Sci. 1191, 201-218. doi: 10.1111/j.1749-6632.2010.05447.x

Buxbaum, L. J., Kyle, K., and Menon, R. (2005). On beyond mirror neurons: Internal representations subserving imitation and recognition of skilled object-related actions in humans. Cogn. Brain Res. 25, 226-239. doi: 10.1016/j.cogbrainres.2005.05.014

Buxbaum, L. J., and Saffran, E. M. (2002). The semantics of object manipulation and object function: a double dissociation. Brain Lang. 82, 179-199. doi: 10.1016/S0093-934X(02)00014-7

Buxbaum, L. J., Schwartz, M. F., and Carew, T. G. (1997). The role of semantic memory in object use. Cogn. Neuropsychol. 14, 219-254. doi: 10.1080/026432997381565

*Cubelli, R., Marchetti, C., Boscolo, G., and Della Sala, S. (2000). Cognition in action: testing a model of limb apraxia. Brain Cogn. 44, 144-165. doi: 10.1006/brcg.2000.1226

*Dawson, A. M., Buxbaum, L. J., and Duff, S. V. (2010). The impact of left hemisphere stroke on force control with familiar and novel objects: Neuroanatomic substrates and relationship to apraxia. Brain Res. 1317, 124-136. doi: 10.1016/j.brainres.2009.11.034 
Della Sala, S., Faglioni, P., Motto, C., and Spinnler, H. (2006). Hemisphere asymmetry for imitation of hand and finger movements, Goldenberg's hypothesis reworked. Neuropsychologia 44, 1496-1500. doi: 10.1016/j.neuropsychologia. 2005.11.011

Dovern, A., Fink, G. R., and Weiss, P. H. (2012). Diagnosis and treatment of upper limb apraxia. J. Neurol. 259, 1269-1283. doi: 10.1007/s00415-011-6336-y

*Flores-Medina, Y., Chávez-Oliveros, M., Medina, L. D., Rodríguez-Agudelo, Y., and Solís-Vivanco, R. (2014). Brain lateralization of complex movement: neuropsychological evidence from unilateral stroke. Brain Cogn. 84, 164-169. doi: 10.1016/j.bandc.2013.11.010

*Foundas, A. L., Macauley, B. L., Raymer, A. M., Maher, L. M., Heilman, K. M., and Gonzalez Rothi, L. J. (1995). Ecological implications of limb apraxia: evidence from mealtime behavior. J. Int. Neuropsychol. Soc. 1, 62-66.

Goldenberg, G. (1995). Imitating gestures and manipulating a manikin. The representation of the human body in ideomotor apraxia.Neuropsychologia 33, 63-72. doi: 10.1016/0028-3932(94)00104-W

Goldenberg, G. (1999). Matching and imitation of hand and finger postures in patients with damage in the left or right hemispheres. Neuropsychologia 37, 559-566. doi: 10.1016/S0028-3932(98)00111-0

Goldenberg, G. (2009). Apraxia and the parietal lobes. Neuropsychologia 47, 1449-1459. doi: 10.1016/j.neuropsychologia.2008.07.014

Goldenberg, G. (2013a). Apraxia - The cognitive side of motor control. Cortex doi: 10.1016/j.cortex.2013.07.016. [Epub ahead of print].

Goldenberg, G. (2013b). Apraxia in left-handers. Brain 136, 2592-2601. doi: $10.1093 /$ brain/awt181

Goldenberg, G., and Hagmann, S. (1997). The meaning of meaningless gestures: a study of visuo-imitative apraxia. Neuropsychologia 35, 333-341. doi: 10.1016/S0028-3932(96)00085-1

*Goldenberg, G., and Hagmann, S. (1998a). Therapy of activities of daily living in patients with apraxia. Neuropsychol. Rehabil. 8, 123-141.

*Goldenberg, G., and Hagmann, S. (1998b). Tool use and mechanical problem solving in apraxia. Neuropsychologia 36, 581-589.

*Goldenberg, G., Hartmann, K., and Schlott, I. (2003). Defective pantomime of object use in left brain damage: apraxia or asymbolia? Neuropsychologia 41, 1565-1573. doi: 10.1016/S0028-3932(03)00120-9

*Goldenberg, G., Hartmann-Schmid, K., Sürer, F., Daumüller, M., and Hermsdörfer, J. (2007). The impact of dysexecutive syndrome on use of tools and technical devices. Cortex 43, 424-435. doi: 10.1016/S0010-9452(08) 70467-2

Goldenberg, G., and Spatt, J. (2009). The neural basis of tool use. Brain 132, 1645-1655. doi: 10.1093/brain/awp080

*Halsband, U., Schmitt, J., Weyers, M., Binkofski, F., Grützner, G., and Freund, H.J. (2001). Recognition and imitation of pantomimed motor acts after unilateral parietal and premotor lesions: a perspective on apraxia. Neuropsychologia 39, 200-216. doi: 10.1016/S0028-3932(00)00088-9

*Hanna-Pladdy, B., Daniels, S. K., Fieselman, M. A., Thompson, K., Vasterling, J. J., Heilman, K. M., et al. (2001). Praxis lateralization: errors in right and left hemisphere stroke. Cortex 37, 219-230. doi: 10.1016/S0010-9452(08) 70569-0

*Hartmann, K., Goldenberg, G., Daumüller, M., and Hermsdörfer, J. (2005). It takes the whole brain to make a cup of coffee: the neuropsychology of naturalistic actions involving technical devices. Neuropsychologia 43, 625-637. doi: 10.1016/j.neuropsychologia.2004.07.015

Heath, M., Roy, E. A., Westwood, D., and Black, S. E. (2001). Patterns of apraxia associated with the production of intransitive limb gestures following left and right hemisphere stroke. Brain Cogn. 46, 165-169. doi: 10.1016/S02782626(01)80057-9

*Heilman, K. M., Maher, L. M., Greenwald, M. L., and Rothi, L. J. G. (1997). Conceptual apraxia from lateralized lesions. Neurology 49, 456-464.

Heilman, K. M., Rothi, L. J. G., Mack, L., Feinberg, T., and Watson, R. T. (1986). Apraxia after a superior parietal lesion. Cortex 22, 141-150. doi: 10.1016/S00109452(86)80038-7

Heilman, K. M., Rothi, L. J., and Valenstein, E. (1982). Two forms of Ideomotor apraxia. Neurology 32, 342-346. doi: 10.1212/WNL.32.4.342

Hermsdörfer, J., Hentze, S., and Goldenberg, G. (2006). Spatial and kinematic features of apraxic movement depend on the mode of execution. Neuropsychologia 44, 1642-1652. doi: 10.1016/j.neuropsychologia.2006.03.023

*Hermsdörfer, J., Li, Y., Randerath, J., Roby-Brami, A., and Goldenberg, G. (2013). Tool use kinematics across different modes of execution.
Implications for action representation and apraxia. Cortex 49, 184-199. doi: 10.1016/j.cortex.2011.10.010

Hodges, J. R., Bozeat, S., Lambon Ralph, M. A., Patterson, K., and Spatt, J. (2000). The role of knowledge in object use: evidence from semantic dementia. Brain 123, 1913-1925. doi: 10.1093/brain/123.9.1913

*Hogrefe, K., Ziegler, W., Weidinger, N., and Goldenberg, G. (2012). Non-verbal communication in severe aphasia: influence of aphasia, apraxia, or semantic processing? Cortex 48, 952-962. doi: 10.1016/j.cortex.2011.02.022

*Jacobs, S., Bussel, B., Combeaud, M., and Roby-Brami, A. (2009). The use of a tool requires its incorporation into the movement: evidence from stick-pointing in apraxia. Cortex 45, 444-455. doi: 10.1016/j.cortex.2007.12.009

*Jarry, C., Osiurak, F., Delafuys, D., Chauviré, V., Etcharry-Bouyx, F., and Le Gall, D. (2013). Apraxia of tool use: more evidence for the technical reasoning hypothesis. Cortex 49, 2322-2333. doi: 10.1016/j.cortex.2013.02.011

*Jax, S. A., Buxbaum, L. J., and Moll, A. D. (2006). Deficits in movement planning and intrinsic coordinate control in ideomotor apraxia. J. Cogn. Neurosci. 18, 2063-2076. doi: 10.1162/jocn.2006.18.12.2063

Kalénine, S., Shapiro, A., and Buxbaum, L. J. (2013). Processing of action means and outcome in the left hemisphere stroke. Neuropsychologia 51, 1224-1233. doi: 10.1016/j.neuropsychologia.2013.03.017

Lesourd, M., Le Gall, D., Baumard, J., Croisile, B., Jarry, C., and Osiurak, F. (2013a). Apraxia and Alzheimer's disease: review and perspectives. Neuropsychol. Rev. 23 234-256. doi: 10.1007/s11065-013-9235-4

Lesourd, M., Le Gall, D., Baumard, J., Croisile, B., Jarry, C., and Osiurak, F. (2013b). Apraxie et maladie d'Alzheimer. Rev. Neuropsychol. 5, 213-222. doi: 10.1684/nrp.2013.0273

*Lunardelli, A., Zadini, A., Gigli, G., and Rumiati, R. I. (2008). Praxic and executive components in tool use learning: the role of imitation. Cogn. Neuropsychol. 25, 1077-1098. doi: 10.1080/02643290801921541

*Neiman, M. R., Duffy, R. J., Belanger, S. A., and Coelho, C. A. (2000). The assessment of limb apraxia: relationship between performances on singleand multiple-object tasks by left hemisphere damaged aphasic subjects. Neuropsychol. Rehabil. 10, 429-448. doi: 10.1080/096020100412005

Osiurak, F. (2013a). Apraxia of tool use is not a matter of affordances. Front. Hum. Neurosci. 7:890. doi: 10.3389/fnhum.2013.00890

Osiurak, F. (2013b). Tool use and manual actions: the human body as a means versus an end. Cortex. doi: 10.1016/j.cortex.2013.10.013. [Epub ahead of print].

Osiurak, F. (2014). What neuropsychology tells us about human tool use? the four constraints theory (4CT): mechanics, space, time, and effort. Neuropsychol. Rev. 24, 88-115. doi: 10.1007/s11065-014-9260-y

*Osiurak, F., Aubin, G., Allain, P., Jarry, C., Etcharry-Bouyx, F., Richard, I., et al. (2008). Different constraints on grip selection in brain-damaged patients: object use versus object transport. Neuropsychologia 46, 2431-2434. doi: 10.1016/j. neuropsychologia.2008.03.018

* Osiurak, F., Jarry, C., Allain, P., Aubin, G., Etcharry-Bouyx, F., Richard, I., et al. (2009). Unusual use of objects after unilateral brain damage. The technical reasoning model.Cortex 45, 769-783. doi: 10.1016/j.cortex.2008. 06.013

Osiurak, F., Jarry, C., and Le Gall, D. (2010). Grasping the affordances. Understanding the reasoning. Toward a dialectical theory of human tool use. Psychol. Rev. 117, 517-540. doi: 10.1037/a0019004

Osiurak, F., Jarry, C., and Le Gall, D. (2011). Re-examining the gesture engram hypothesis. New perspectives on apraxia of tool use.Neuropsychologia 49, 299-312. doi: 10.1016/j.neuropsychologia.2010.12.041

Osiurak, F., Jarry, C., Lesourd, M., Baumard, J., and Le Gall, D. (2013). Mechanical problem solving strategies in left-brain damaged patients with apraxia of tool use. Neuropsychologia 51, 1964-1972. doi: 10.1016/j.neuropsychologia.2013. 06.017

*Papeo, L., Negri, G. A. L., Zadini, A., and Rumiati, R. I. (2011). Action performance and action-word understanding: evidence of double dissociations in left-damaged patients. Cogn. Neuropsychol. 27, 428-461. doi: 10.1080/02643294. 2011.570326

*Poole, J. L., Sadek, J., and Haaland, K. Y. (2011). Meal preparation abilities after left or right hemisphere stroke. Arch. Phys. Med. Rehabil. 92, 590-596. doi: 10.1016/j.apmr.2010.11.021

*Randerath, J., Goldenberg, G., Spijkers, W., Li, Y., and Hermsdörfer, J. (2010). Different left brain regions are essential for grasping a tool compared with its subsequent use. Neuroimage 53, 171-180. doi: 10.1016/j.neuroimage.2010. 06.038 
*Randerath, J., Goldenberg, G., Spijkers, W., Li, Y., and Hermsdörfer, J. (2011). From pantomime to actual tool use: how affordances can facilitate actual tooluse. Neuropsychologia 49, 2410-2416. doi: 10.1016/j.neuropsychologia.2011. 04.017

Riddoch, M. J., Humphreys, G. W., and Price, C. J. (1989). Routes to action: evidence from apraxia. Cogn. Neuropsychol. 6, 437-454. doi: 10.1080/02643298908 253424

Rothi, L. J. G., Ochipa, C., and Heilman, K. M. (1991). A cognitive neuropsychological model of limb praxis. Cogn. Neuropsychol. 8, 443-458. doi: $10.1080 / 02643299108253382$

*Roy, E. A., Black, S. E., Blair, N., and Dimeck, P. T. (1998). Analyses of deficits in gestural pantomime. J. Clin. Exp. Neuropsychol. 20, 628-643.

Roy, E. A., and Hall, C. (1992). "Limb apraxia. A process approach," in Vision and Motor Control, eds P. Luc and E. Digby (Amsterdam: Elsevier), 261-282. doi: 10.1016/S0166-4115(08)62018-X

* Roy, E. A., Heath, M., Westwood, D., Schweizer, T. A., Dixon, M. J., Black, S. E., et al. (2000). Tasks demands and limb apraxia in stroke. Brain Cogn. 44, 253-279. doi: 10.1006/brcg.2000.1230

Roy, E. A., and Square, P. A. (1985). "Common considerations in the study of limb, verbal and oral apraxia," in Neuropsychological Studies of Apraxia and Related Disorders, ed E. A. Roy (Amsterdam: Elsevier), 111-161.

Rumiati, R. I. (2005). Right, left or both? Brain hemispheres and apraxia of naturalistic actions. Trends Cogn. Sci. 9, 167-169. doi: 10.1016/j.tics.2005.02.007

*Schnider, A., Hanlon, R. E., Alexander, D. N., and Benson, D. F. (1997). Ideomotor apraxia: behavioral dimensions and neuroanatomical basis. Brain Lang. 58, 125-136.

Schwartz, M. F., Buxbaum, L. J., Montgomery, M. W., Fitzpatrick-Desalme, E. J., Hart, T., Ferraro, M., et al. (1999). Naturalistic action production following right hemisphere stroke. Neuropsychologia 37, 51-66. doi: 10.1016/S00283932(98)00066-9

Silveri, M. C., and Ciccarelli, N. (2009). Semantic memory in object use. Neuropsychologia 47, 2634-2641. doi: 10.1016/j.neuropsychologia.2009.05.013

* Stamenova, V., Roy, E. A., and Black, S. (2010). Associations and dissociations of transitive and intransitive gestures in left and right hemisphere stroke patients. Brain Cogn. 72, 483-490. doi: 10.1016/j.bandc.2010.01.004

*Vanbellingen, T., Kersten, B., van Hemelrijk, B., van de Winckel, A., Bertschi, M., Müri, R., et al. (2010). Comprehensive assessment of gesture production: a new test of upper limb apraxia (TULIA). Eur. J. Neurol. 17, 59-66. doi: 10.1111/j.1468-1331.2009.02741.x

Conflict of Interest Statement: The authors declare that the research was conducted in the absence of any commercial or financial relationships that could be construed as a potential conflict of interest.

Received: 05 March 2014; accepted: 01 May 2014; published online: 21 May 2014. Citation: Baumard J, Osiurak F, Lesourd M and Le Gall D (2014) Tool use disorders after left brain damage. Front. Psychol. 5:473. doi: 10.3389/fpsyg.2014.00473

This article was submitted to Cognition, a section of the journal Frontiers in Psychology.

Copyright (c) 2014 Baumard, Osiurak, Lesourd and Le Gall. This is an openaccess article distributed under the terms of the Creative Commons Attribution License (CC BY). The use, distribution or reproduction in other forums is permitted, provided the original author(s) or licensor are credited and that the original publication in this journal is cited, in accordance with accepted academic practice. No use, distribution or reproduction is permitted which does not comply with these terms.

\footnotetext{
${ }^{*}$ Data displayed in tables and figures were extracted from references preceded by a $*$.
} 